\title{
Generalized Synchronization of Different Chaotic Systems Based on Nonnegative Off-Diagonal Structure
}

\author{
Ling Guo, ${ }^{1,2}$ Xiaohong Nian, ${ }^{2}$ and Huan $\operatorname{Pan}^{3}$ \\ ${ }^{1}$ College of Electrical Engineering, Northwest University for Nationalities, Lanzhou 730030, China \\ ${ }^{2}$ College of Information Science and Engineering, Central South University, Changsha 410075, China \\ ${ }^{3}$ College of Physics Electrical Information Engineering, Ningxia University, Yinchuan 750021, China
}

Correspondence should be addressed to Ling Guo; gling0826@126.com

Received 31 May 2013; Accepted 12 September 2013

Academic Editor: Jian Guo Zhou

Copyright (C) 2013 Ling Guo et al. This is an open access article distributed under the Creative Commons Attribution License, which permits unrestricted use, distribution, and reproduction in any medium, provided the original work is properly cited.

\begin{abstract}
The generalized synchronization problem is studied in this paper for different chaotic systems with the aid of the direct design method. Based on Lyapunov stability theory and matrix theory, some sufficient conditions guaranteeing the stability of a nonlinear system with nonnegative off-diagonal structure are obtained. Then the control scheme is designed from the stable system by the direct design method. Finally, two numerical simulations are provided to verify the effectiveness and feasibility of the proposed method.
\end{abstract}

\section{Introduction}

As a very important topic in nonlinear science, chaos synchronization has been studied extensively in both theory and applications, such as mathematics, physics, biology, and engineering community. Since the pioneering work of $[1,2]$ in 1990, many techniques have been successfully applied to the synchronization of chaotic systems, for example, the timedelay feedback control, adaptive control, backstepping control, sliding mode control, linear state feedback control, and nonlinear control. Moreover, a variety of concepts of synchronization has also been proposed including complete synchronization $[3,4]$, phase synchronization [5], lag synchronization [6], antisynchronization $[7,8]$, projective synchronization $[9,10]$, cluster synchronization [11-13], and generalized synchronization [14-19].

Generalized synchronization can be regarded as an extension of complete synchronization, antisynchronization, or projective synchronization and it means that there exists a functional relation between the states of two systems. It is noted that the two systems may have different dimensions. The generalized synchronization problem between two complex networks with nonlinear coupling and time-varying delay was developed in [18]. Two variants of generalized synchronization between strictly different chaotic systems were proposed in [19]. Reference [20] presented a new scheme to achieve generalized synchronization between different discrete-time chaotic (hyperchaotic) systems. Reference [21] investigated the problem of chaos synchronization between two different uncertain chaotic systems with input nonlinearities. Acharyyaa and Amritkarb have briefly discussed some recent developments in the study of generalized synchronization in [22].

Recently, the direct design method $[3,10]$ is proposed to investigate synchronization of chaotic systems. It presents an easy procedure for selecting proper controllers and the simple controller deriving from this method is easy to implement. The idea of this method is to transform the original error system into a stable system under a control scheme. It is clear that the structure of stable system plays a key role in this method. Liu et al. introduced the direct design method and realized synchronization of chaotic systems based on the stable system with tridiagonal structure [3]. Subsequently, Cai et al. studied generalized projective synchronization of chaotic systems based on the stable system with antisymmetric structure [10]. In this paper, the direct design method is developed to address generalized synchronization of different chaotic systems. The main contribution is to design a nonlinear control scheme by means of matrix theory. The error dynamic system is changed into a stable system with a special structure which is different from the existing results in $[3,10]$. 


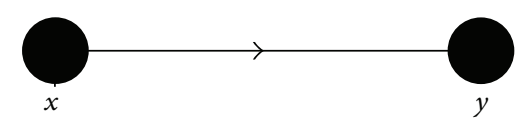

FIGURE 1: Schematic diagram of a drive-response configuration. Here, the system $x$ is driving the system $y$.

The rest of this paper is organized as follows. In Section 2, the problem is formulated under investigation. The main result is given in Section 3. The stable system with nonnegative off-diagonal structure is discussed and a control scheme is presented by the direct design method. In Section 4, numerical simulations are shown to illustrate and verify the effectiveness of proposed theoretical results. The paper is concluded in the last section.

The following notations are used throughout this paper. $R^{n}$ denotes the $n$ dimensional Euclidean space. $R^{n \times n}$ is the set of all $n \times n$ real matrices. For a vector $x=\left[x_{1}, x_{2}, \ldots, x_{n}\right]^{T} \in$ $R^{n}, x^{T}$ represents the transpose of the vector $x,\|x\|=$ $\left(x^{T} x\right)^{1 / 2}$, and $x>0$ if all entries $x_{i}$ are positive. $\rho(\bullet)$ is the spectral radius of matrix $\bullet$.

\section{Problem Description and Preliminaries}

In this section, generalized synchronization between two coupled chaotic systems is introduced. The concept of generalized synchronization was first proposed for unidirectionally coupled systems, that is, systems coupled in a drive response configuration, by Rulkov et al. [14] (see Figure 1). Consider a system in the form of

$$
\dot{x}=f(x),
$$

where $x=\left[x_{1}, x_{2}, \ldots, x_{m}\right]^{T} \in R^{m}$ is the state vector, $f(\cdot)$ : $R^{m} \rightarrow R^{m}$ is a continuous function. Equation (1) is regarded as a drive system. Then the controlled response system is described by

$$
\dot{y}=A y+g(y)+u,
$$

where $y=\left[y_{1}, y_{2}, \ldots, y_{n}\right]^{T} \in R^{n}, A \in R^{n \times n}$ is a state matrix, $g(\cdot): R^{n} \rightarrow R^{n}$ is a nonlinear continuous function, and $u$ is referred to a control scheme to be designed later.

Definition 1 (see [16]). Given a vector map $\varphi: R^{m} \rightarrow R^{n}$, if systems (1) and (2) satisfy

$$
\lim _{t \rightarrow \infty}\|y-\varphi(x)\|=0,
$$

then we say that system (1) achieves generalized synchronization with system (2).

Define the error variable $e=y-\varphi(x)$, where $\varphi$ is continuously differentiable; then the dynamical error system is written as

$$
\begin{aligned}
\dot{e}= & A y+g(y)-D \varphi(x) f(x)+u \\
= & A e+g(e+\varphi(x))+A \varphi(x) \\
& -D \varphi(x) f(x)+u,
\end{aligned}
$$

where $D \varphi(x)$ is the Jacobian matrix of the map $\varphi(x)$; that is,

$$
D \varphi(x)=\left[\begin{array}{cccc}
\frac{\partial \varphi_{1}(x)}{\partial x_{1}} & \frac{\partial \varphi_{1}(x)}{\partial x_{2}} & \cdots & \frac{\partial \varphi_{1}(x)}{\partial x_{m}} \\
\frac{\partial \varphi_{2}(x)}{\partial x_{1}} & \frac{\partial \varphi_{2}(x)}{\partial x_{2}} & \cdots & \frac{\partial \varphi_{2}(x)}{\partial x_{m}} \\
\vdots & \vdots & \ddots & \vdots \\
\frac{\partial \varphi_{n}(x)}{\partial x_{1}} & \frac{\partial \varphi_{n}(x)}{\partial x_{2}} & \cdots & \frac{\partial \varphi_{n}(x)}{\partial x_{m}}
\end{array}\right]
$$

In fact, system (1) achieving generalized synchronization with system (2) is equivalent to the asymptotical stability of the error system (4) at $e=0$. Hence, the essential problem here is how to choose a control scheme $u$ to make system (4) asymptotically stable at the origin.

Some necessary results $[23,24]$ in matrix theory are needed to derive our main results.

Lemma 2. Matrix $Q$ is a nonsingular M-matrix under one of the following conditions, and the conditions are all equivalent:

(i) the eigenvalues of $Q$ have positive real parts;

(ii) there exists a positive diagonal matrix $S$ such that QS + $S Q^{T}$ is strictly diagonally dominant and hence also positive definite.

Lemma 3. For any matrix $Q \in \mathscr{Z}$, the following statements are equivalent to each other:

(i) $Q$ is a singular M-matrix with "Property c";

(ii) there exists a symmetric positive definite matrix $W$ such that $W Q+Q^{T} W$ is positive semidefinite; that is, matrix $-Q$ is Lyapunov stable.

Lemma 4. If $Q \in \mathscr{Z}$ and if there exists vector $\Gamma>0$ such that $\mathrm{Q} \Gamma \geq 0$, then $\mathrm{Q}$ is a singular M-matrix with "Property c."

\section{Main Results}

In this section, we focus on the stability of a nonlinear system with nonnegative off-diagonal structure. Then a control scheme is proposed by the direct design method.

Consider the following system with state dependent coefficients described by

$$
\dot{z}=D(z) z
$$

where $z=\left[z_{1}, z_{2}, \ldots, z_{n}\right]^{T} \in R^{n}$ is the state vector, and $D(z)=\left[d_{i j}(z)\right] \in R^{n \times n}, i, j=1,2, \ldots, n$, is the coefficient matrix.

Theorem 5. If the $D(z)=\left[d_{i j}(z)\right] \in R^{n \times n}, i, j=1,2, \ldots, n$, satisfies the following conditions:

$$
\begin{aligned}
& \text { (i) } d_{i j}(z) \geq 0, i \neq j ; \\
& \text { (ii) }-d_{i i}(z)>\sum_{j \neq i} d_{j i}(z) \text {; }
\end{aligned}
$$

then system (6) is asymptotically stable. 
Proof. Construct a Lyapunov function

$$
V(z)=z^{T} P_{1} z
$$

where $P_{1}$ is a positive diagonal matrix. The time derivative of $V(z)$ along the trajectories of (6) is

$$
\begin{aligned}
\dot{V}(z) & =z^{T} P_{1} \dot{z}+\dot{z}^{T} P_{1} z \\
& =z^{T}\left(P_{1} D+D^{T} P_{1}\right) z .
\end{aligned}
$$

If $P_{1} D+D^{T} P_{1}$ is negative definite, it is clear that the equilibrium $z=0$ of system (6) is asymptotically stable from Lyapunov stability theory. For a nonsingular $M$-matrix $-D^{T}$, the existence of such a matrix $P_{1}$ is guaranteed by Lemma 2. The proof of Theorem 5 is completed.

Theorem 6. If the $D(z)=\left[d_{i j}(z)\right] \in R^{n \times n}, i, j=1,2, \ldots, n$, satisfies the following conditions:

(i) $d_{i j}(z) \geq 0, i \neq j$;

(ii) there exists a vector $\Gamma>0$, such that $D(z) \Gamma \leq 0$,

and the invariant set of system (6) only includes the origin, then system (6) is asymptotically stable.

Proof. Construct a Lyapunov function

$$
V(z)=z^{T} P_{2} z
$$

where $P_{2}$ is a symmetric positive definite matrix. Then the time derivative of $V(z)$ is

$$
\begin{aligned}
\dot{V}(z) & =z^{T} P_{2} \dot{z}+\dot{z}^{T} P_{2} z \\
& =z^{T}\left(P_{2} D+D^{T} P_{2}\right) z .
\end{aligned}
$$

If $P_{2} D+D^{T} P_{2}$ is negative semidefinite and the invariant set of system (6) only includes the origin, the equilibrium $z=0$ of system (6) is asymptotically stable. It follows from conditions (i) and (ii) and Lemma 4 that $-D$ is a singular $M$-matrix with "Property c." Lemma 3 guarantees the existence of matrix $P_{2}$. Then the proof of Theorem 6 is completed.

Our main purpose is to design a control scheme $u$ in (2) by the direct design method. The system (4) with this control scheme can be transformed into the form of system (6), that is, $\dot{e}=D e$, which is asymptotically stable at the origin according to the aforementioned conclusions. The result can be presented as follows.

Theorem 7. If $-D \varphi(x)$ is an $M$-matrix, system (1) achieves generalized synchronization with system (2) under the nonlinear control scheme

$$
u=(D \varphi(x)-A) e-g(e+\varphi(x))-A \varphi(x)+D \varphi(x) f(x) .
$$

Proof. Substituting (11) into error system (4), the following equation is obtained:

$$
\dot{e}=D \varphi(x) e .
$$

From Theorem 5 or Theorem 6 , we conclude that if $-D \varphi(x)$ is an $M$-matrix, system (12) is asymptotically stable about zero. The proof of Theorem 7 is completed.

Remark 8 . The results obtained in the above discussion can be applied to complete synchronization [3], antisynchronization, and generalized projective synchronization [10] of chaotic systems, since complete synchronization and generalized projective synchronization are special cases of generalized synchronization.

Remark 9. It is worth noticing that the structure of matrix $D \varphi(x)$ in this paper is different from the existing results in $[3,10]$. So in this sense, compared with the work of $[3,10]$, the results of this paper will give us more freedom in designing the controller.

Remark 10. The control scheme (11) is designed from the stable system by the direct design method. It is worth noting that the direct design approaches transform the original systems into the system with stable tridiagonal structure by inputs. The direct design method can design the controllers with simpler structure than backstepping for nonlinear systems with low dimension.

\section{Numerical Examples}

In this section, to illustrate the effectiveness of the proposed method, we discuss two numerical simulations for two cases: (i) for identical systems, generalized projective synchronization between two Chua's circuits; (ii) for different dimensional systems, generalized synchronization between HyperRössler system and Chen system.

4.1. Generalized Projective Synchronization of Chua's Circuit. Consider the following Chua's circuit

$$
\begin{gathered}
\dot{x}_{1}=\sigma\left(x_{2}-x_{1}^{3}-r x_{1}\right), \\
\dot{x}_{2}=x_{1}-x_{2}+x_{3}, \\
\dot{x}_{3}=-h x_{2}
\end{gathered}
$$

as the drive system and the controlled response system is given in the following form:

$$
\begin{gathered}
\dot{y}_{1}=\sigma\left(y_{2}-y_{1}^{3}-r y_{1}\right)+u_{1}, \\
\dot{y}_{2}=y_{1}-y_{2}+y_{3}+u_{2}, \\
\dot{y}_{3}=-h y_{2}+u_{3},
\end{gathered}
$$

where $x=\left[x_{1}, x_{2}, x_{3}\right]^{T}, y=\left[y_{1}, y_{2}, y_{3}\right]^{T}$ are the state variables, $\sigma=10, h=16$, and $r=-0.143$.

Generalized projective synchronization of systems (13) and (14) will be realized with $\varphi(x)=\alpha x$, where $\alpha$ is a nonzero constant and referred to as a scaling factor. Defining the error 


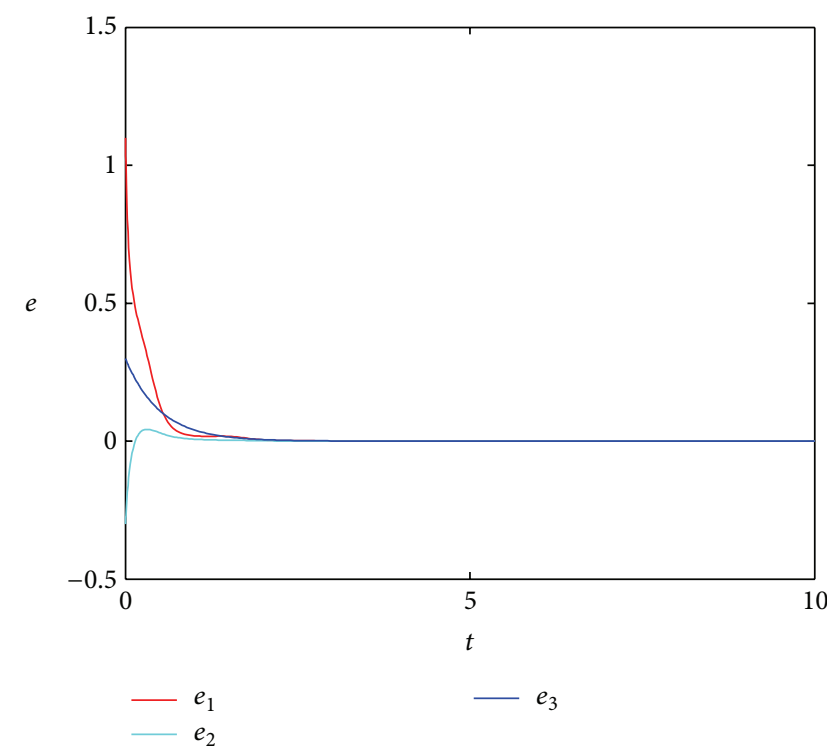

(a)

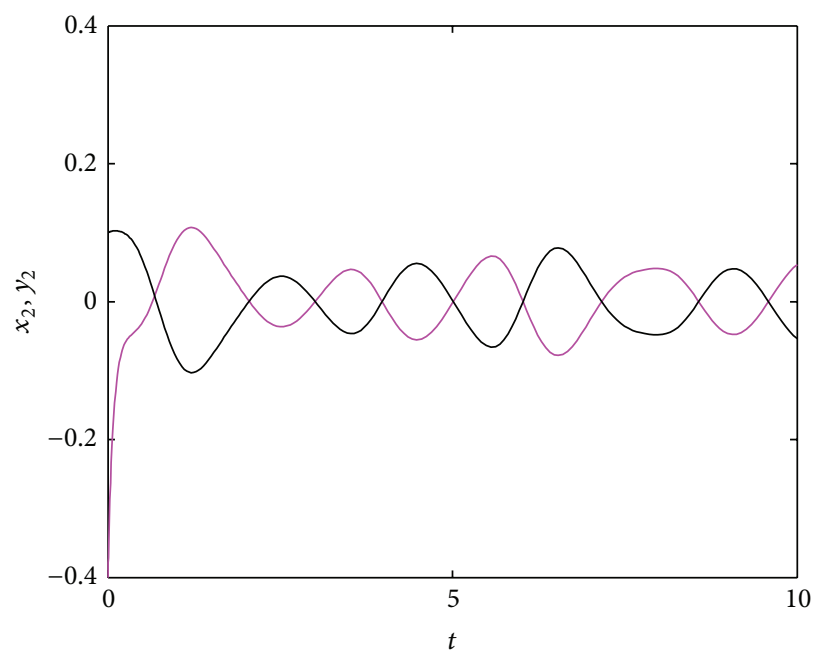

(c)

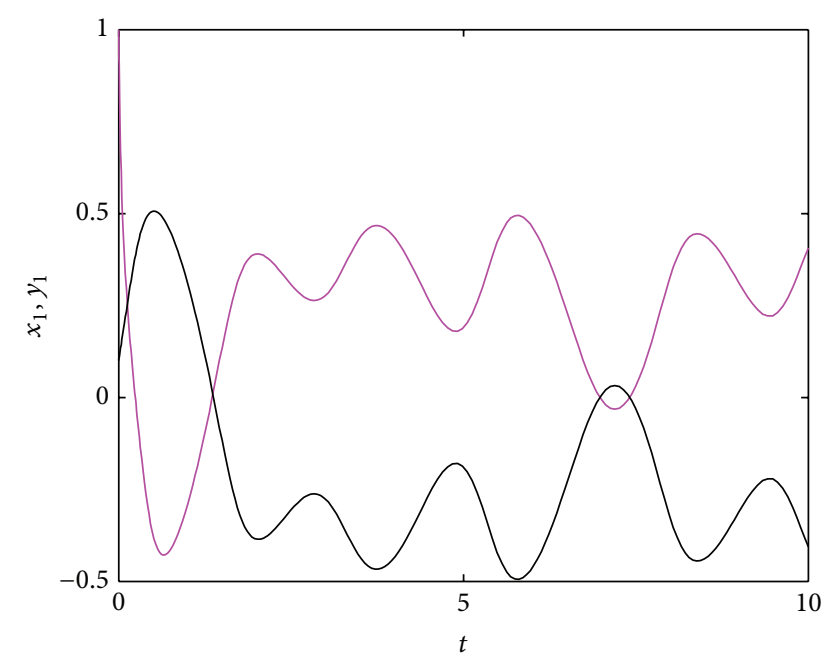

(b)

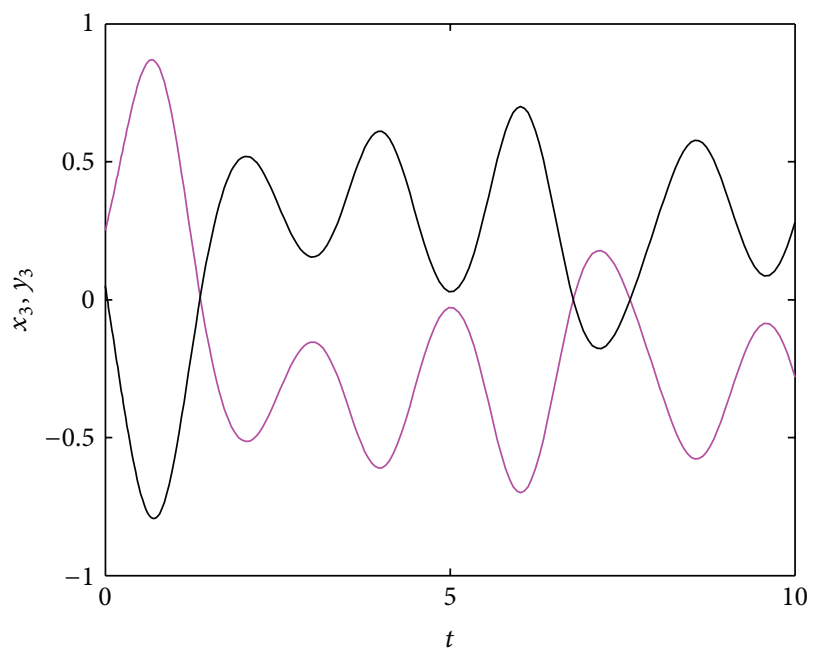

(d)

Figure 2: Antisynchronization errors and state trajectories of systems (13) and (14) under the controller (16).

vector $e=\left[e_{1}, e_{2}, e_{3}\right]^{T}$, where $e_{i}=y_{i}-\alpha x_{i}, i=1,2,3$, the error system can be written as

$$
\begin{aligned}
\dot{e}= & {\left[\begin{array}{ccc}
1.43-30\left(\alpha x_{1}+0.5 e_{1}\right)^{2}-7.5 e_{1}^{2} & 10 & 0 \\
1 & -1 & 1 \\
0 & -16 & 0
\end{array}\right] e } \\
& +\left[\begin{array}{c}
10 x_{1}^{3}\left(1-\alpha^{2}\right) \\
0 \\
0
\end{array}\right]+\left[\begin{array}{l}
u_{1} \\
u_{2} \\
u_{3}
\end{array}\right] .
\end{aligned}
$$

Next, the two following controllers are designed from Theorems 5 and 6 , respectively.
At first, design the controller as

$$
\begin{aligned}
& u_{1}=1.43 e_{1}-2 e_{1}-10 x_{1}^{3}\left(1-\alpha^{2}\right), \\
& u_{2}=-10 e_{2}, \quad u_{3}=16 e_{2}-2 e_{3} .
\end{aligned}
$$

Substitute the controller (16) into (15) and get

$$
\dot{e}=B_{1} e,
$$

where

$$
B_{1}=\left[\begin{array}{ccc}
-30\left(\alpha x_{1}+0.5 e_{1}^{2}\right)^{2}-7.5 e_{1}^{2}-2 & 10 & 0 \\
1 & -11 & 1 \\
0 & 0 & -2
\end{array}\right] .
$$




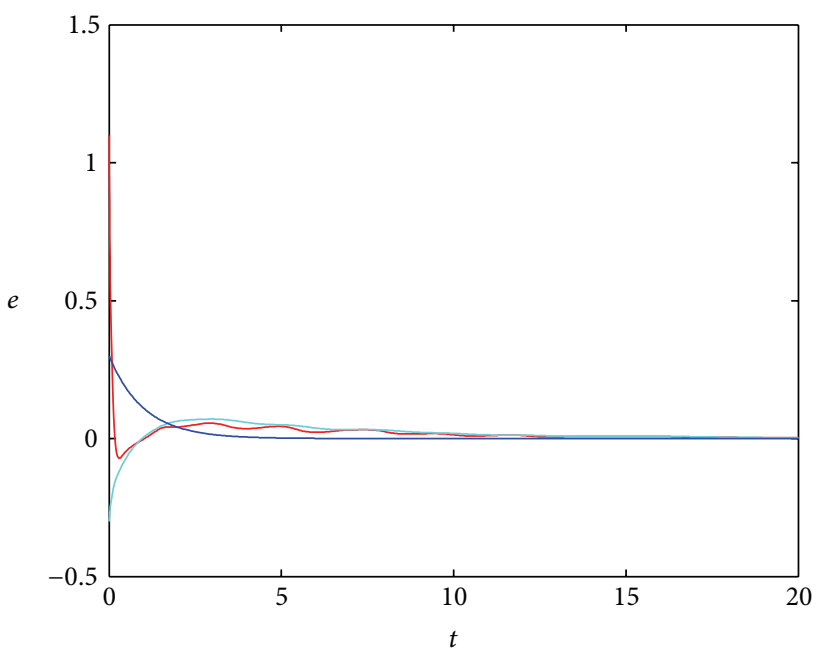

$-e_{1}$

(a)

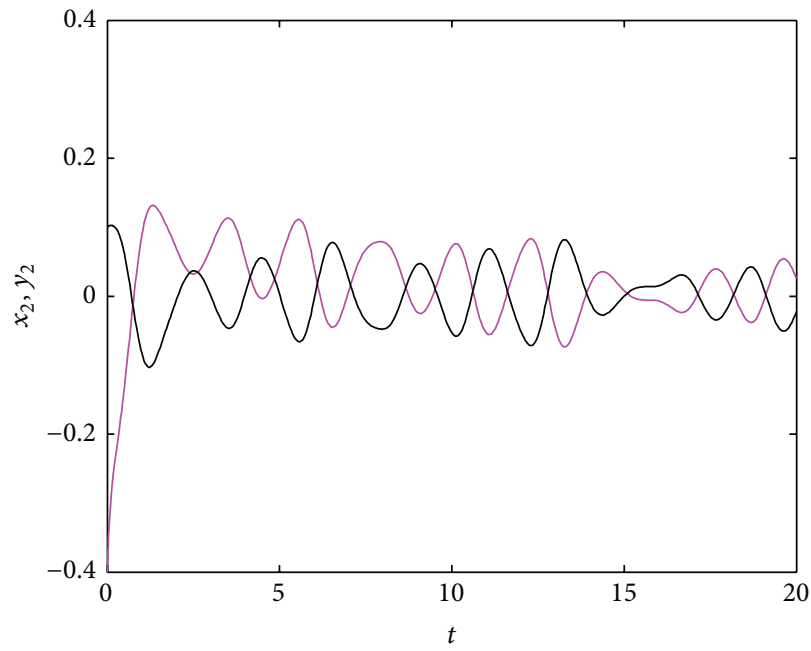

(c)

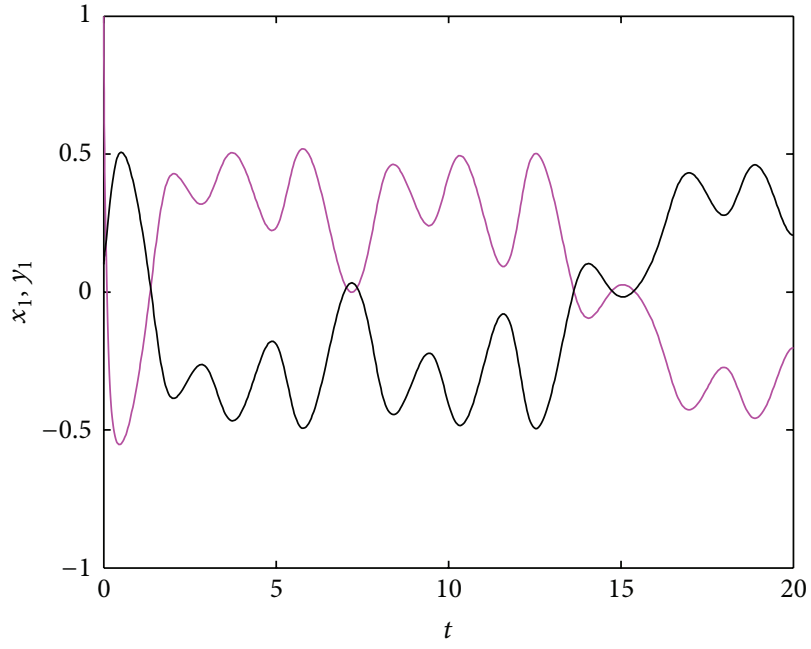

(b)

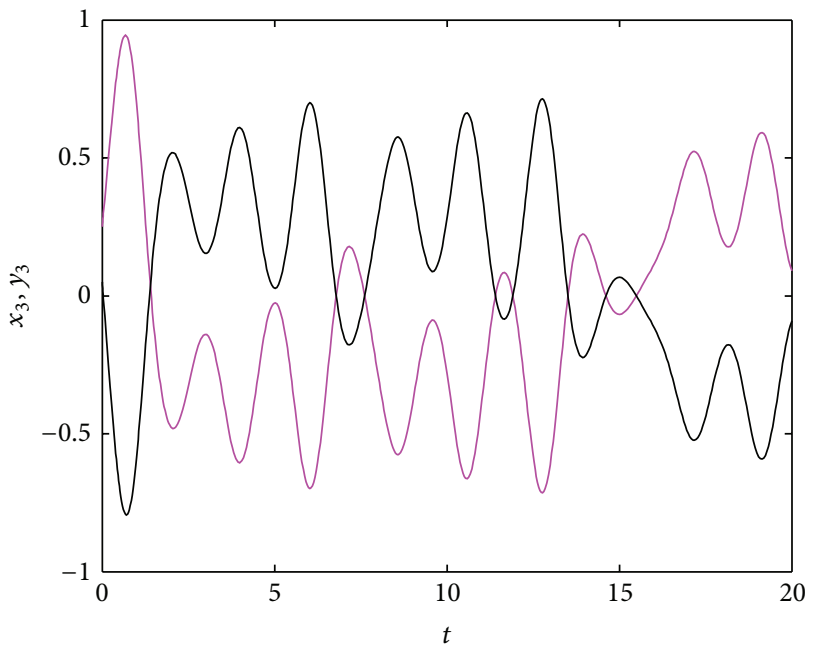

(d)

Figure 3: Antisynchronization errors and state trajectories of systems (13) and (14) under the controller (19).

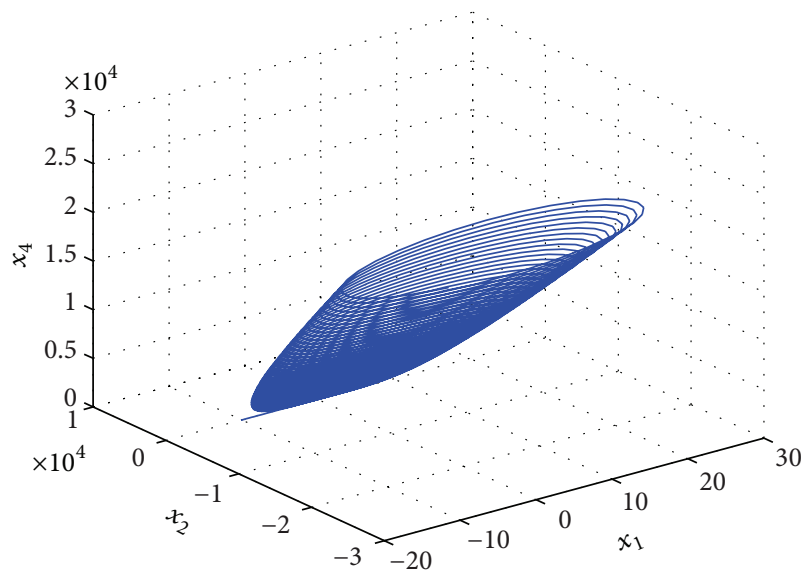

FIgURE 4: Chaotic attractor in $\left(x_{1}, x_{2}, x_{4}\right)$ space of HyperRössler system with parameters $a=0.25, b=0.05$, and $c=3$ and initial state $x(0)=[-10,1,2,0]^{T}$.

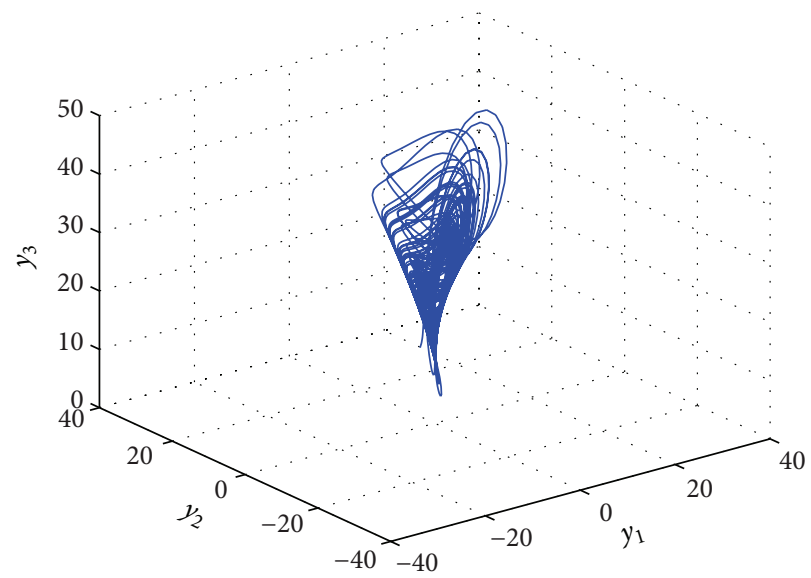

FIGURE 5: Chaotic attractor of Chen system with parameters $\gamma=28$, $\beta=35$, and $\eta=3$ and initial state $y(0)=[3,8,10]^{T}$. 


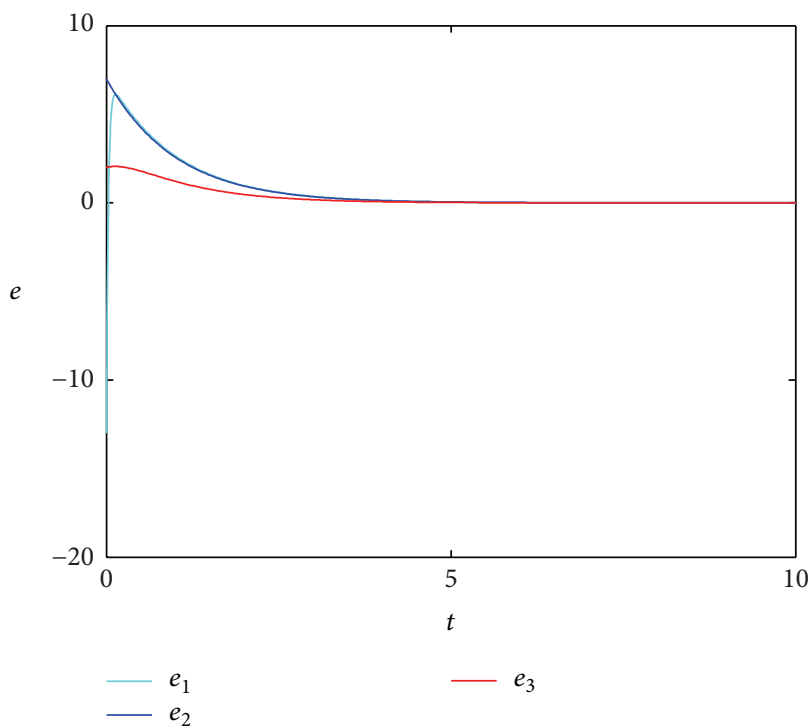

(a)

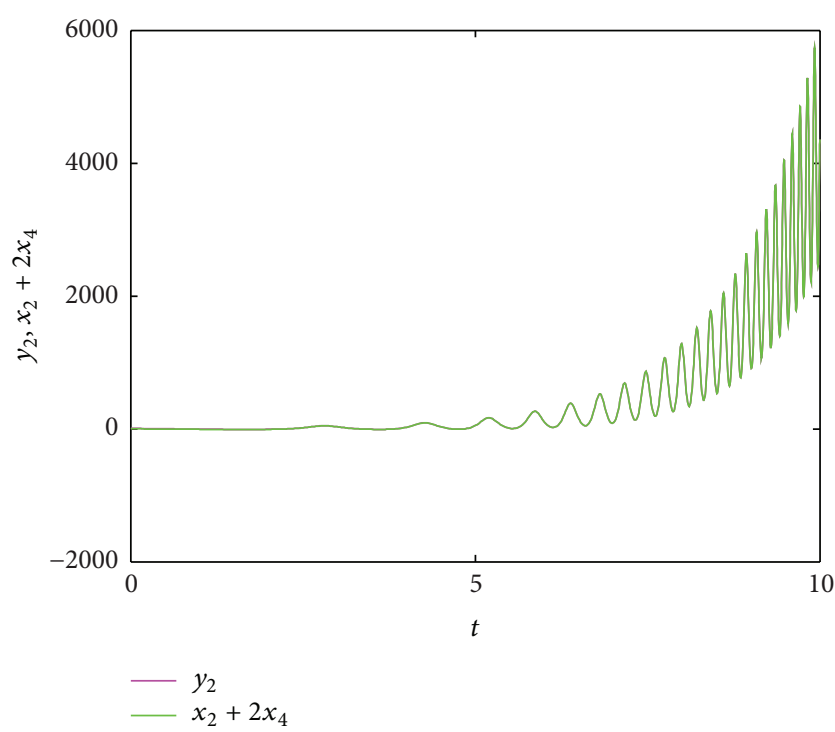

(c)

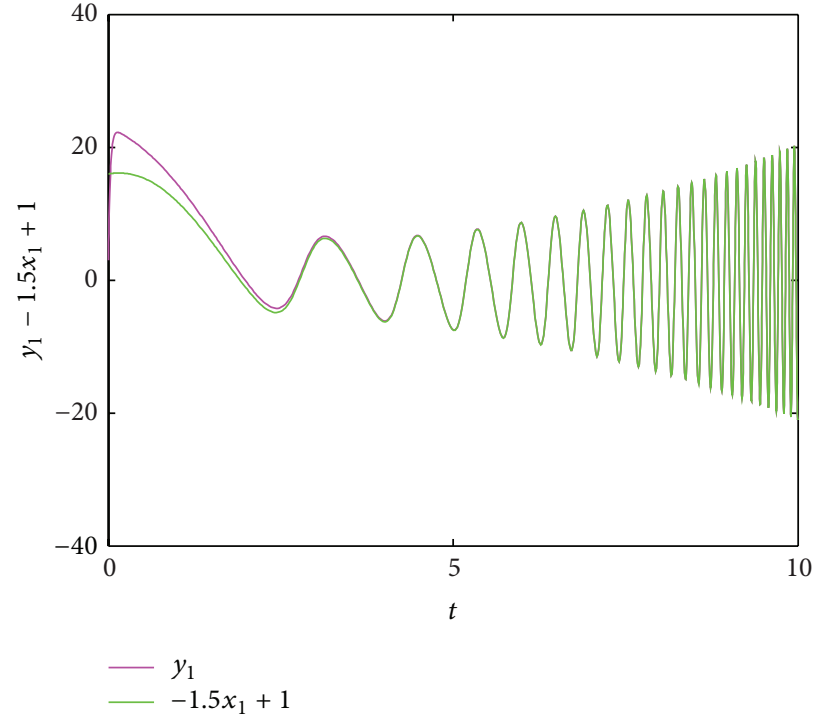

(b)

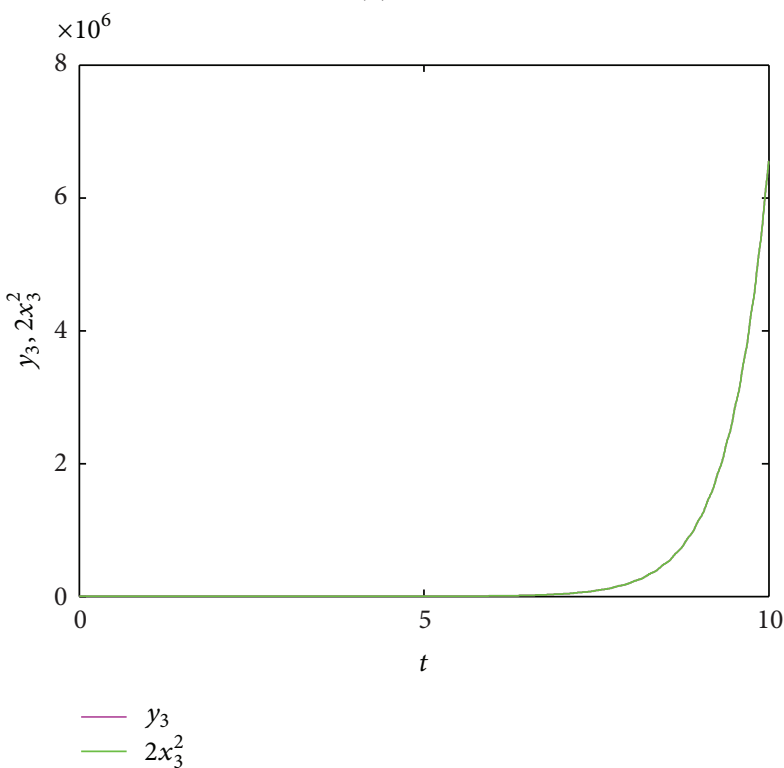

(d)

FIGURE 6: Generalized synchronization errors and state trajectories of systems (22) and (23) under the controller (26).

It is easy to verify that all the conditions of Theorem 5 are satisfied for system (17). Therefore, system (17) is asymptotically stable at the origin. Namely, system (13) achieves generalized synchronization with system (14) under the controller (16).

Secondly, design the controller

$$
\begin{gathered}
u_{1}=1.43 e_{1}-10 e_{1}-10 x_{1}^{3}\left(1-\alpha^{2}\right), \\
u_{2}=0, \quad u_{3}=16 e_{2}-e_{3} .
\end{gathered}
$$

Then the error system (15) is changed into the following system:

$$
\dot{e}=B_{2} e,
$$

where

$$
B_{2}=\left[\begin{array}{ccc}
-30\left(\alpha x_{1}+0.5 e_{1}^{2}\right)^{2}-7.5 e_{1}^{2}-10 & 10 & 0 \\
1 & -1 & 1 \\
0 & 0 & -1
\end{array}\right] .
$$

It is noted that condition (ii) of Theorem 6 is satisfied for (20) by choosing $\Gamma=[9.2,10,0.5]^{T}$. According to Theorem 6 , system (20) is asymptotically stable at the origin. Hence, system (13) achieves generalized synchronization with system (14) under the controller (19).

The initial states of drive and response systems (13) and (14) are $x(0)=[0.1,0.1,0.05]^{T}$ and $y(0)=[1,-0.4,0.25]^{T}$. Numerical simulations for anti-synchronization of systems 
(13) and (14) (i.e., the scaling factor $\alpha=-1$ ) under controllers (16) and (19) are shown in Figures 2 and 3, respectively.

4.2. Generalized Synchronization between HyperRössler System and Chen System. Consider the HyperRössler system

$$
\begin{gathered}
\dot{x}_{1}=-x_{2}-x_{4}, \\
\dot{x}_{2}=x_{1}+a x_{2}+x_{3}, \\
\dot{x}_{3}=b x_{3}-2 a x_{4}, \\
\dot{x}_{4}=c+x_{1} x_{4}
\end{gathered}
$$

as drive system and the controlled response system is the following Chen system:

$$
\begin{gathered}
\dot{y}_{1}=\beta\left(y_{2}-y_{1}\right)+u_{1}, \\
\dot{y}_{2}=(\gamma-\beta) y_{1}-y_{1} y_{3}+\gamma y_{2}+u_{2}, \\
\dot{y}_{3}=y_{1} y_{2}-\eta y_{3}+u_{3},
\end{gathered}
$$

where $x=\left[x_{1}, x_{2}, x_{3}, x_{4}\right]^{T}, y=\left[y_{1}, y_{2}, y_{3}\right]^{T}$ are the state variables, $a=0.25, b=0.05, c=3, \gamma=28, \beta=35$, and $\eta=3$.

Set

$$
\varphi(x)=\left[-1.5 x_{1}+1, x_{2}+2 x_{4}, 2 x_{3}^{2}\right]^{T} .
$$

Defining the error variable $e=y-\varphi(x)$, it follows from (22)(24) that

$$
\begin{aligned}
\dot{e}= & {\left[\begin{array}{ccc}
-35 & 35 & 0 \\
-7-e_{3}-2 x_{3} & 28 & 1.5 x_{1}-1 \\
e_{2}+x_{2}+2 x_{4} & 1-1.5 x_{1} & -3
\end{array}\right] e } \\
& +\left[\begin{array}{c}
52.5 x_{1}+33.5 x_{2}+68.5 x_{4}-35 \\
9.5 x_{1}+27.75 x_{2}-3 x_{3}+56 x_{4}-13+x_{1}\left(3 x_{3}-2 x_{4}\right) \\
\left(x_{2}+2 x_{4}\right)\left(1-1.5 x_{1}\right)-x_{3}\left(0.1 x_{3}-x_{4}+6\right)
\end{array}\right] \\
& +\left[\begin{array}{c}
u_{1} \\
u_{2} \\
u_{3}
\end{array}\right] .
\end{aligned}
$$

Design the controller

$$
\begin{aligned}
u_{1}= & -52.5 x_{1}-33.5 x_{2}-68.5 x_{4}+35 \\
u_{2}= & \left(7+e_{3}+2 x_{3}\right) e_{1}-29 e_{2}+\left(1-1.5 x_{1}\right) e_{3} \\
& -9.5 x_{1}-27.75 x_{2}+3 x_{3}-56 x_{4}+13 \\
& -x_{1}\left(3 x_{3}-2 x_{4}\right) \\
u_{3}= & -\left(e_{2}+x_{2}+2 x_{4}\right) e_{1}+1.5 x_{1} e_{2} \\
& -\left(x_{2}+2 x_{4}\right)\left(1-1.5 x_{1}\right)+x_{3}\left(0.1 x_{3}-x_{4}+6\right) .
\end{aligned}
$$

Then the error system (25) is rewritten as the following system:

$$
\dot{e}=B_{3} e,
$$

where

$$
B_{3}=\left[\begin{array}{ccc}
-35 & 35 & 0 \\
0 & -1 & 0 \\
0 & 1 & -3
\end{array}\right]
$$

It is clear that all conditions of Theorem 5 and Theorem 6 are satisfied for system (25), where condition (ii) of Theorem 6 can be verified by choosing $\Gamma=[1,1,1]^{T}$. According to Theorem 5 or Theorem 6 , system (25) is asymptotically stable at the origin. Hence, system (22) achieves generalized synchronization with system (23) under the controller (26).

Chaotic attractors of HyperRössler system and Chen system with initial states $x(0)=[-10,1,2,0]^{T}$ and $y(0)=$ $[3,8,10]^{T}$ are shown in Figures 4 and 5 , respectively. Numerical simulation for generalized synchronization of systems (22) and (23) under controller (26) is shown in Figure 6.

\section{Conclusions}

This paper has studied generalized synchronization of different chaotic systems. By using Lyapunov stability theory and matrix theory, the stability of a nonlinear system with nonnegative off-diagonal structure is obtained. Then the control scheme can be designed from transforming the error system into this stable system by using the direct design method. It is worth noticing that the structure of this stable system is significantly different from existing results of the direct design method. Numerical simulations are presented for identical systems and nonidentical systems with different dimension, respectively.

\section{Acknowledgments}

This work is supported by the National Natural Science Foundation of China (61075065, 60774045, U1134108), the Ph.D. Programs Foundation of Ministry of Education of China (20110162110041), and the Talent Introduction Scientific Research Foundation of Northwest University for Nationalities (Grant no. xbmuyjrc201304).

\section{References}

[1] E. Ott, C. Grebogi, and J. A. Yorke, "Controlling chaos," Physical Review Letters, vol. 64, no. 11, pp. 1196-1199, 1990.

[2] L. M. Pecora and T. L. Carroll, "Synchronization in chaotic systems," Physical Review Letters, vol. 64, no. 8, pp. 821-824, 1990.

[3] B. Liu, Y. Zhou, M. Jiang, and Z. Zhang, "Synchronizing chaotic systems using control based on tridiagonal structure," Chaos, Solitons and Fractals, vol. 39, no. 5, pp. 2274-2281, 2009.

[4] Z. S. Duan, G. R. Chen, and L. Huang, "Synchronization of weighted networks and complex synchronized regions," Physics Letters A, vol. 372, no. 21, pp. 3741-3751, 2008.

[5] M. G. Rosenblum, A. S. Pikovsky, and J. Kurths, "Phase synchronization of chaotic oscillators," Physical Review Letters, vol. 76, no. 11, pp. 1804-1807, 1996.

[6] S. Taherion and Y. C. Lai, "Observability of lag synchronization of coupled chaotic oscillators," Physical Review E, vol. 59, no. 6, pp. R6247-R6250, 1999. 
[7] J. B. Liu, C. F. Ye, S. J. Zhang, and W. T. Song, "Anti-phase synchronization in coupled map lattices," Physics Letters A, vol. 274, no. 1-2, pp. 27-29, 2000.

[8] Y. Zhang and J. Sun, "Chaotic synchronization and anti-synchronization based on suitable separation," Physics Letters A, vol. 330, no. 6, pp. 442-447, 2004.

[9] R. Mainieri and J. Rehacek, "Projective synchronization in three-dimensional chaotic systems," Physical Review Letters, vol. 82, no. 15, pp. 3042-3045, 1999.

[10] N. Cai, Y. W. Jing, and S. Y. Zhang, "Generalized projective synchronization of different chaotic systems based on antisymmetric structure," Chaos, Solitons and Fractals, vol. 42, no. 2, pp. 1190-1196, 2009.

[11] W. Wu, W. J. Zhou, and T. P. Chen, "Cluster synchronization of linearly coupled complex networks under pinning control," IEEE Transactions on Circuits and Systems I, vol. 56, no. 4, pp. 829-839, 2009.

[12] W. G. Xia and M. Cao, "Clustering in diffusively coupled networks," Automatica, vol. 47, no. 11, pp. 2395-2405, 2011.

[13] L. Guo, X. H. Nian, Y. Zhao, and Z. S. Duan, "Cluster synchronisation of Lur'e dynamical networks," IET Control Theory \& Applications, vol. 6, no. 16, pp. 2499-2508, 2012.

[14] N. F. Rulkov, M. M. Sushchik, L. S. Tsimring, and H. D. I. Abarbanel, "Generalized synchronization of chaos in directionally coupled chaotic systems," Physical Review E, vol. 51, no. 2, pp. 980-994, 1995.

[15] L. Kocarev and U. Parlitz, "Generalized synchronization, predictability, and equivalence of unidirectionally coupled dynamical systems," Physical Review Letters, vol. 76, no. 11, pp. 18161819, 1996.

[16] S. S. Yang and C. K. Duan, "Generalized synchronization in chaotic systems," Chaos, Solitons and Fractals, vol. 9, no. 10, pp. 1703-1707, 1998.

[17] G. Zhang, Z. R. Liu, and Z. J. Ma, "Generalized synchronization of different dimensional chaotic dynamical systems," Chaos, Solitons and Fractals, vol. 32, no. 2, pp. 773-779, 2007.

[18] Q. X. Bian and H. X. Yao, "Generalized synchronization between two complex dynamical networks with time-varying delay and nonlinear coupling," Mathematical Problems in Engineering, vol. 2011, Article ID 978612, 15 pages, 2011.

[19] J. G. Barajas Ramírez, K. P. Cuéllar Galarza, and R. Femat, “Generalized synchronization of strictly different systems: partialstate synchrony," Chaos, Solitons and Fractals, vol. 45, no. 3, pp. 193-204, 2012.

[20] G. Grassi, "Generalized synchronization between different chaotic maps via dead-beat control," Chinese Physics B, vol. 21, no. 5, pp. 1-7, 2012.

[21] M. P. Aghababa and A. Heydari, "Chaos synchronization between two different chaotic systems with uncertainties, external disturbances, unknown parameters and input nonlinearities," Applied Mathematical Modelling, vol. 36, no. 4, pp. 1639-1652, 2012.

[22] S. Acharyya and R. E. Amritkar, "Generalized synchronization of coupled chaotic systems," The European Physical Journal, vol. 222, no. 3-4, pp. 939-952, 2013.

[23] Z. H. Qu, Cooperative Control of Dynamical Systems: Applications to Autonomous Vehicles, Springer, London, UK, 2009.

[24] A. Berman and R. J. Plemmons, Nonnegative Matrices in the Mathematical Sciences, Academic Press, New York, NY, USA, 1979. 


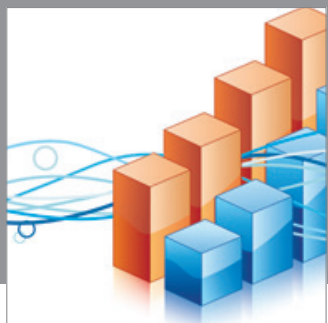

Advances in

Operations Research

mansans

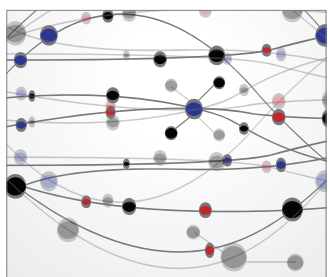

The Scientific World Journal
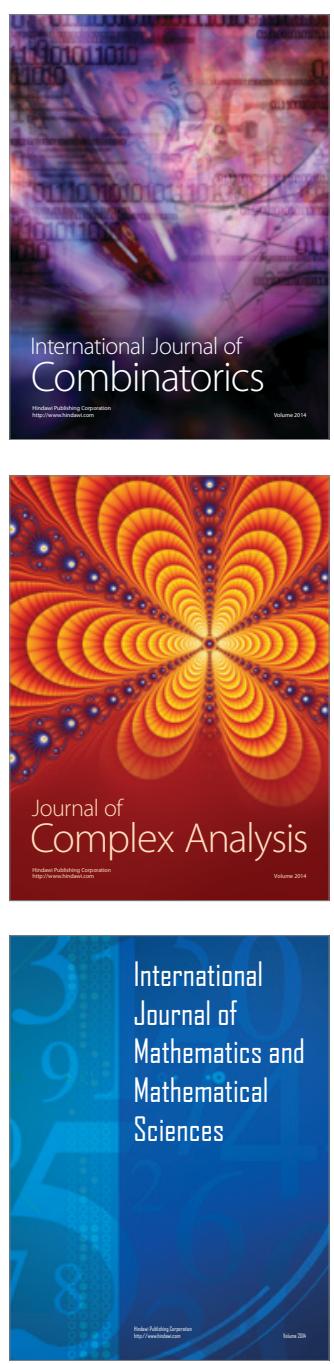
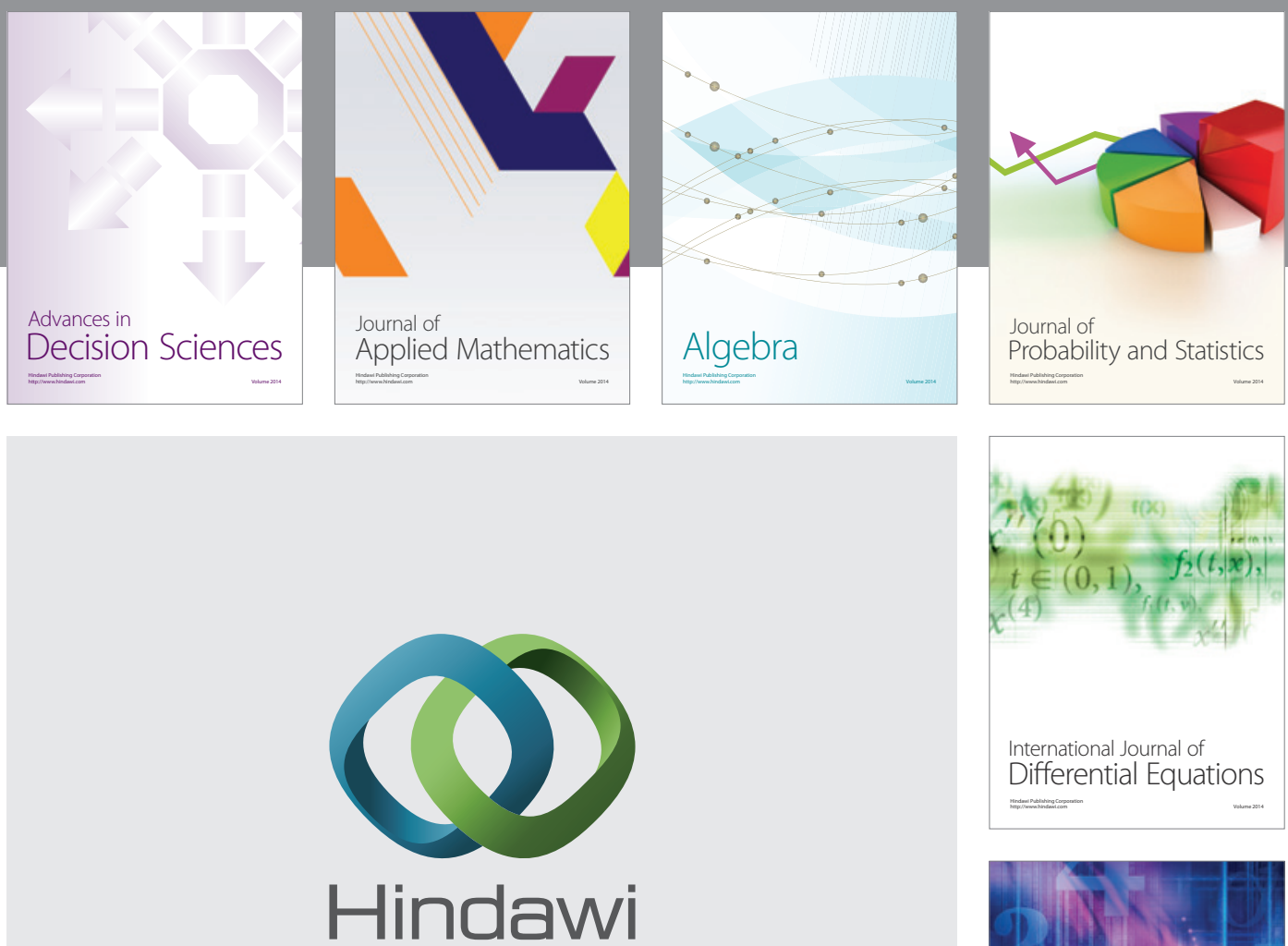

Submit your manuscripts at http://www.hindawi.com
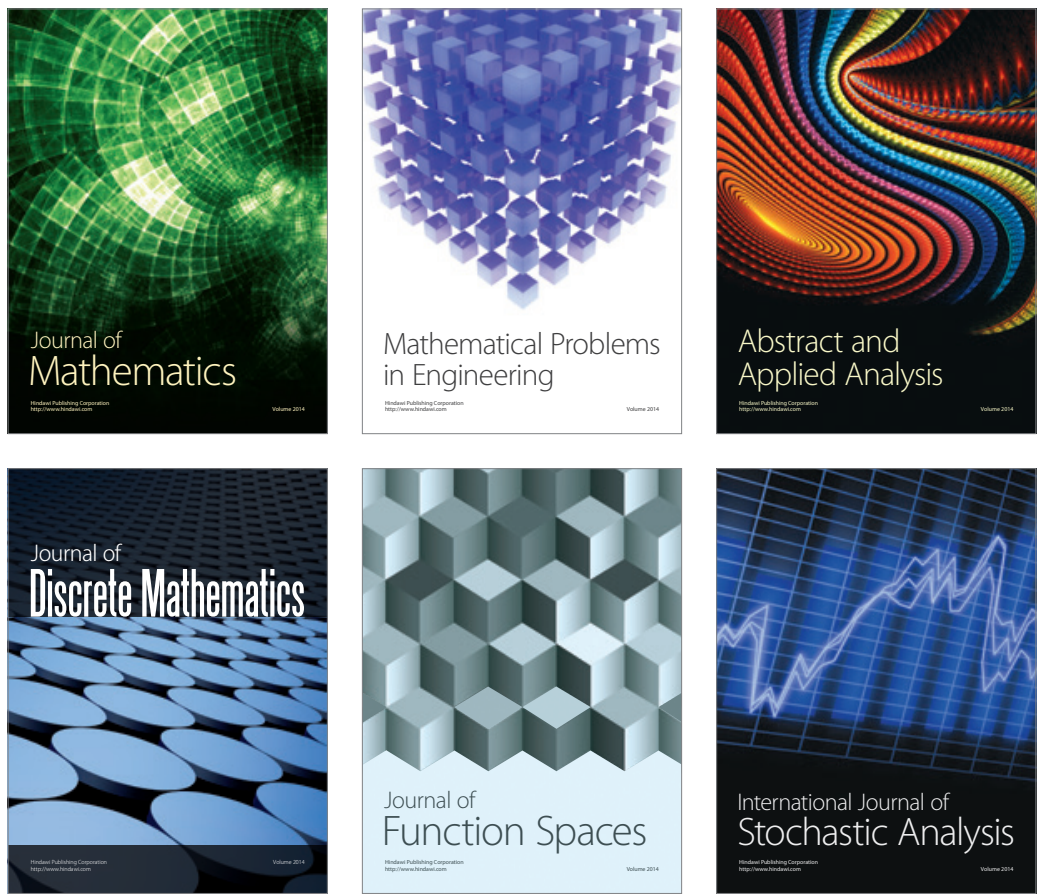

Journal of

Function Spaces

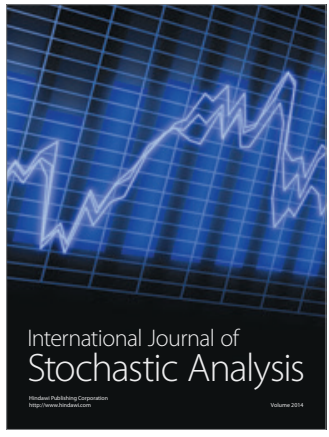

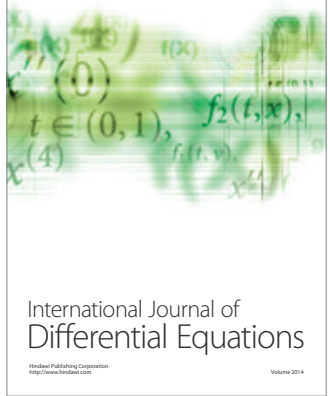
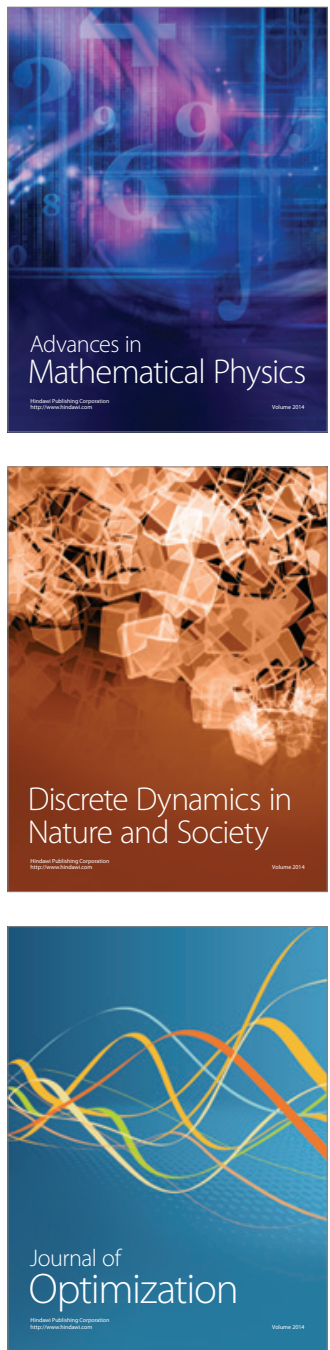